\title{
Physical Clearing Mechanisms in Power Industry
}

\author{
Karla Atkins \\ Basic and Applied \\ Simulation Science Group \\ Los Alamos National Laboratory \\ Los Alamos, NM 87545 \\ Email: karla@lanl.gov
}

\author{
Christopher M. Homan \\ Dept. of Computer Science \\ Rochester Institute of Technology \\ Rochester, NY 14623 \\ Email: cmh@cs.rit.edu
}

\author{
Achla Marathe \\ Modeling, Algorithms \\ and Informatics Group \\ Los Alamos National Laboratory \\ Los Alamos, NM 87545 \\ Email: achla@lanl.gov
}

\begin{abstract}
This paper studies the performance of several different physical clearing mechanisms in the electricity market. The study is done using the simulation tool, Marketecture, developed at the Los Alamos National Laboratory. Two cities, Portland and Chicago, are used for simulating the physical clearing algorithms. The algorithms were chosen according to their ability to serve as a proxy for some of the well known policies. Our results show that, based on the economic measures chosen, both cities perform very similarly under the different physical clearing mechanisms.
\end{abstract}

\section{INTRODUCTION AND MOTIVATION}

The electricity literature provides abundant information on issues related to the deregulation of energy sector and its impact on generation of electricity, market power, market efficiency, consumption, etc. See [4], [3], [5], [15], [22], [24], [11], [12], [5], [9], [14], [16], [17], [13], [7], [23] and the references therein. However there have been relatively fewer papers addressing the issues of transmission network constraints, physical clearing and the optimal use of the electrical grid. This study aims to fill such a gap by analyzing the impact of different physical clearing mechanisms in a bilateral market. Deregulation has been designed to decouple the controllers of the network from the power producers, making it difficult to regulate the levels of power on the network. In practice, deregulation is complicated by the fact that all power companies have to share the same power network and the network's capacity is limited. To overcome these problems, most U.S. states have set up an ISO (independent system operator): a non-profit governing body to arbitrate the use of the network. The basic questions facing ISOs are how to decide which contracts to deny (due to capacity constraints), and who is to bear the costs accrued when contracts are denied. Several criteria/policies have been proposed and/or are being legislated by the states as possible guidelines for the ISO to select a maximum-sized subset of contracts that can be cleared simultaneously [21]. These include: (a) Minimum Flow Denied: The ISO selects the subset of contracts that denies the least amount of proposed power flow. This proposal favors clearing bigger contracts first. (b) First-in First-out: The contract that comes first gets cleared first; this is the least discriminating to the contractors. (c) Maximum Consumers Served: This clears the smallest contracts first and favors the small buyers whose interests tend to go unheard.

There are three key issues in deciding policies that entail specific mechanisms for selecting a subset of contracts: fairness of a given policy to producers and consumers; the computational complexity of implementing a policy, and how sound a given policy is from an economic standpoint. Here we focus on evaluating the efficacy of a given policy from an economic point of view. It is intuitively clear that the underlying network, its capacity and topology, and the spatial locations of the bilateral contracts on the network, will play an important role in determining the efficacy of these policies. The computational complexity issue of these policies has been discussed in a companion paper. For details refer to [2]. The work reported here is done as part of the "Marketecture" project at the Los Alamos National Laboratory. Marketecture is a simulation based analysis tool for analyzing deregulated power markets.

We carry out an experimental analysis of several algorithms for physically clearing the contracts made in the bilateral market. The algorithms were chosen according to their ability to serve as a proxy for some of the above-stated policies. The algorithms are as follows: The Descending-Order Heuristic is a proxy for the Minimum Flow Denied policy. The ASCENDINGORder Heuristic serves as a proxy for the Maximum Contracts Served policy. The RANDOM-ORDER HEURISTIC clears the contracts in the random order. This algorithm was chosen as a proxy for the Firstin First-out policy. Such a policy is probably the most natural clearing mechanism and is currently in place at many exchanges.

The rest of this paper is organized as follows. In Section II, we discuss the model and the methodology used. In Section 3 we describe the power grid. Section 
4 shows the results and analysis of the Portland and Chicago study. The final section concludes the paper.

\section{The Model And Methodology}

We have developed an agent based, microscopic and highly scalable model to study electricity markets. The model together with the simulation based analytical system is called MARKetecture. Marketecture is an end-to-end simulation tool. It is connected to the urban infrastructure as well as the physical electrical grid. The urban infrastructure drives the individualistic, location and time dependent, demographics based power demand profiles of a synthetic population. Section 2.2 explains this in more detail. These demand profiles are aggregated to wholesale levels and are used to bid in the bilateral and other markets. The market model is connected to the physical grid (a power flow module) which allows the ISO to check the feasibility of the economically cleared bilateral contracts.

Marketecture offers three different kinds of markets; poolco, bilateral and spot market. For more details on "Marketecture" project see [1]. For the purpose of this study, we will focus on the bilateral market. In a bilateral market, buyers and suppliers pair off independently to negotiate the economic terms of the contracts. All contracts are submitted to ISO for a feasibility check. The ISO runs the power flow solver to determine if the electrical grid has enough transmission capacity to support the physical terms of the contract.

\section{A. Normative Representation of Individual Entities}

Each type of entity in the simulator (e.g., market, consumer, supplier, etc.) has a set of parameters associated with it. These parameters give each entity its unique character. Understanding how the simulator works essentially means understanding how the parameters of each entity are affected by the other entities in the system. The list below describes the main entities in the system, and defines their attributes, using notation of the form: for an entity $e$ having attribute $a$, we will use the "." operator to denote the value of $a$. For instance, given two Consumers $c_{1}, c_{2}$ and a bus $u ; c_{2} . u$ refers to $c_{2}$ 's BUS and $c_{1} . u$ refers to $c_{1}$ 's BUS.

- The power grid is a graph $G=(V, E), V$ is the set of busses or substations, where generators inject power into or consumers draw power from the grid and $E$ is the set of power lines that connect one bus to another.

- For $c=$ Consumer, $c . L$ is a list of Locations that the Consumer represents. $c . b$ is the BUYER who represents the CONSUMER in the BILATERALMARKET. $c . u$ is the BUS from which $c$ draws power and c. $f_{\text {demand }}(\cdot)$ is his demand function.

- For $b=$ BUYER, represents a collection of CONSUMERS and makes all economic decisions (with respect to the power market) on its constituent CONSUMERS' behalf. Each BUYER has a list of the Consumers $C=\left\{c_{1}, \ldots, c_{n}\right\}$, that the buyer represents.

- For $g=$ Generator, produces electricity and is represented by a SELLER $g . S$ in the market. $g . u$ is the bus that connects $g$ to the power grid. $g . f_{\text {cost }}(\cdot)$ is its cost function. $g . q_{a s k}$ is the quantity of power that the $g$ 's SELLER plans to sell in the market.

For $s=$ SELLER, represents a collection of GENERATORS, $G=\left\{g_{1}, \ldots, g_{n}\right\}$, and makes all economic decisions (with respect to the power markets) on its constituent GENERATORS' behalf.

\section{B. Demand Profiles for Portland and Chicago Popula-} tion

We spend significant effort in simulating realistic demand profiles on an hourly basis for each consumer for the cities of Portland and Chicago. We then aggregate these demand profiles to a location level which get further aggregated to a buyer, who owns the locations and trades in the bilateral market on behalf of his consumers and locations. Marketecture interfaces with an urban population mobility simulator (UPMoST), developed at the Los Alamos National Laboratory. The UPMoST simulator provides the demographic information as well as the activity and physical location of each consumer for the synthetic population of Portland and Chicago. ${ }^{1}$ This allows us to compute power demand profile for each consumer, at any given time, based on his location, activity and demographic information. For further details on UPMoST and its interface with Marketecture see [18] and [1].

\section{The Bilateral Market}

Running the bilateral market requires pairing of SELLERS and BUYERS who can negotiate a contract with each other. The following bind event allows a random matching of the SELLERS and BUYERS with a userdefined probability.

1) Bind Event: The bind event is where SELLERS and BUYERS are matched for an upcoming runBilateralMarket event. The syntactical form that bind event can take is the following:

\section{bind random num}

where num is a floating point number in $[0,1]$ provided by the user. In this case the algorithm is

\footnotetext{
Algorithm BIND

${ }^{1}$ Synthetic consumers are people from a synthetic population. Our synthetic population is an imitation of the real population of Portland and Chicago. The synthetic population preserves the key features of the real population and has the same statistical properties such as correlation structure, joint distributions of the demographic variables, spatial distribution of households etc.
} 
Let $M=\emptyset$.

Foreach (BUyeR, SELLER) $(b, s)$,

Let $r$ be a floating point number sampled uniformly from $[0,1]$;

If $r \leq$ num,

$$
\text { Let } M=M \cup\{(b, s)\} \text {; }
$$

2) Algorithm for Bilateral Market: Before a runBilateralMarket event occurs, we assume that a bind event has occurred. The RUNBILATERALMARKET algorithm takes one argument, the set of matchings $M$ created by the most recent bind event.

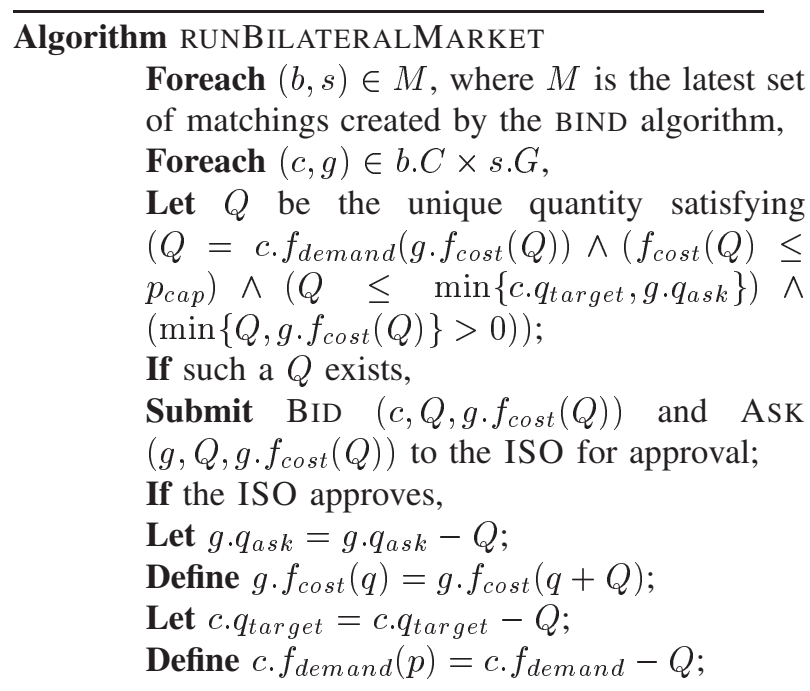

The above algorithm is specified for contract group of 1 , however, this study applies modified algorithm that allows for adjustable buffering of contract groups so that contracts can be submitted to the grid in different batches. Note that the order in which the (BUYER, SELLER) pairs are chosen to negotiate contracts is significant. Because the cost and demand functions are updated as soon as a contract is cleared, as a rule of thumb, contracts that clear earlier will be for more power. The running time of this algorithm is $\Theta\left(\mathrm{C} \cdot \mathrm{G} \cdot n^{2}\right)$, where $\mathrm{C}$ is the number of CONSUMERS, $\mathrm{G}$ is the number of GENERATORs, and $n$ is the number of BUSSES.

\section{Scale of the Study}

The table below shows the scale of this study in terms of the consumers, buildings, generators, substations and transmission lines used for Portland and Chicago.

$\begin{array}{ccc} & \text { Portland } & \text { Chicago } \\ \text { Consumers } & 1.6 \text { million } & 9 \text { million } \\ \text { Locations/Bldg. } & 243,000 & 1.2 \text { million } \\ \text { Generators } & 40 & 157 \\ \text { Transmission Lines } & 718 & 2340 \\ \text { Substations } & 624 & 1078\end{array}$

\section{THE POWER GRID}

In our model, the power grid is a graph $G=(V, E)$, $V$ is the set of busses or substations which are used by the generators to inject power into or by the consumers to draw power from the grid. $E$ is the set of power lines that connect one bus to another. We associate with each bus its location in space. Power lines have two parameters: the amount of power currently flowing through the line and the maximum amount of power that may flow through the line. A real power grid is of course much more complex than our model. For instance, in a real power grid, power is delivered as alternating current (AC). We assume that power is direct current (DC) and can be controlled to any degree of precision. We also do not directly account for resistance in our model. Nonetheless, the model is adequate for our purposes, i.e., to study how congestion on the electrical power grid affects market prices. A resistive network imposes additional constraint on the network and requires solving a quadratic program. Our model provides a worst case scenario since the solutions provided by a resistive network clearing would also encompass the solutions provided by a non-resistive network. The nonresistive network provides an upper bound in terms of the performance of the variables measured here.

\section{A. Feasibility Check}

Access to the power grid is controlled by the independent systems operator (ISO). The ISO is responsible for clearing potential contracts physically or informing the generators and consumers in the market that no such clearing is possible. The ISO also knows the current loading of the grid, which specifies the amount of power load at each substation (bus). The ISO updates the load at each bus where the potential contracts either consume or inject power. With this update, the power flow code is run to check whether the addition of the new loads violates the grid constraints. If no grid constraints are violated, then the contracts are said to clear. Otherwise, they are rejected.

In the bilateral market, the ISO is sent contracts between pairs of consumers and generators. Each contract has a single quantity of power $q$ that the generator must provide to the consumer and a price $p$ that the power costs per unit. Thus, before the power flow algorithm is run, $s_{j}$ of the bus $b_{j}$ where the generator is connected to the grid and $d_{k}$ of the bus $b_{k}$ where the consumer is connected, are both increased by $q$ and $m_{j}$ is set to $s_{j}$. 
With these changes in place, the ISO runs the power flow algorithm. If it finds a solution, the contracts are cleared. For bilateral contracts, this means that each contract is cleared at the price $p$ that was negotiated in the contract. If the algorithm finds no solution, then the values $s_{j}, m_{j}$, and $d_{k}$ are all set to the values they were at before the preceding contracts were sent to the ISO.

\section{B. Load Assignment to Substations}

Given the input data set for our synthetic population, we aggregate demand at each spatial location at which a consumer activity occurs and map it to a unique substation on the power grid. This substation is the closest substation for this location. The total power load at a substation is the sum of the power demands at each location mapped to that substation.

Let $L=\left\{l_{1}, l_{2}, \ldots, l_{i}, \ldots\right\}$ be the set of locations. Let $x\left(l_{i}\right)$ and $y\left(l_{i}\right)$ denote the easting and northing coordinates (in UTM, see [20], [6]) of $l_{i}$ for each $i$. Let $\Sigma=\left\{\sigma_{1}, \sigma_{2}, \ldots, \sigma_{j}, \ldots\right\}$ be the set of substations. Let $x\left(\sigma_{j}\right)$ and $y\left(\sigma_{j}\right)$ denote the easting and northing coordinate (in UTM) of $\sigma_{j}$ for each $j$. We map each location $l_{i}$ to the closest substation where the distance is the Euclidean distance between two points. (The Euclidean distance between the UTM projections of two points approximates the geodesic distance between the points on the surface of the earth.) In other words, if $\sigma\left(l_{i}\right)$ denotes the index of the substation to which location $l_{i}$ is mapped, then

$$
\sigma\left(l_{i}\right)=\underset{j}{\operatorname{argmin}}\left\{\left(x\left(l_{i}\right)-x\left(\sigma_{j}\right)\right)^{2}+\left(y\left(l_{i}\right)-y\left(\sigma_{j}\right)\right)^{2}\right\}
$$

We can compute this map in $O(|\Sigma| \log |\Sigma|+|L| \log |\Sigma|)$ time and $O(|\Sigma|)$ space using a standard point location data structure for the Voronoi diagram of the substations [10].

\section{Results AND AnAlysis}

This study analyzes the performance of the bilateral market under different physical clearing algorithms and different groupings of the bilateral contracts. As mentioned earlier, we use three heuristics for physical clearing. The economically cleared contracts (i) are sorted in descending order and sent to the electrical grid for physical clearing (the largest contract is sent first), (ii) are sorted in the ascending order and sent to the grid for physical clearing (the smallest goes first) and (iii) are sent in random order to the grid for clearing.

For each order, we divide the contracts into different sizes of groups. The groups are in sets of 10, 20, 30, $40,50,60,70,80,90,100$. The reason for trying out different grouping sizes is to see how often should the ISO run the power flow to determine which contracts are feasible. The intuition is that for smaller group sizes, a higher number of contracts can clear but it would be computationally more expensive to run the power flow frequently. On the other hand, if the ISO waits to collect several bilateral contracts before running the power flow, fewer contracts might clear. ISO might use such a simulation to decide which set size is optimal in terms of the variables that matter from a policy point of view. The goal of this study is to carry out such an analysis and see the performance of different physical clearing orders and group sizes. We perform this study on two cities; Portland, Oregon and Chicago, Illinois.

As we know, contracts and volume cleared on the grid heavily depend upon the location of each contract. The congestion on the grid depends not only on the source and sink location of each contract but also the volume and direction of the flow. A higher number of contracts on the grid do not necessarily lead to more congestion. If some contracts are moving power in the reverse direction of congestion, having more contracts can lead to lower congestion. For example, if contract 1 requires moving $100 \mathrm{MW}$ of power from node A (source node) to node B (sink node) but the capacity of the transmission line (between A and B) is only 50MW, contract 1 will not be able to clear. However, if another contract, say, contract 2 is to move $50 \mathrm{MW}$ from node $\mathrm{B}$ to node $\mathrm{A}$, both contract 1 and 2 will clear. This is because $50 \mathrm{MW}$ flow would cancel out as two contracts try to move power in the opposite direction. This study tries to understand this non linear nature of the physical clearing and its dependence upon the topology of the grid and other electrical characteristics of the grid. Below we see the performance of different physical clearing algorithms and contract sets with respect to the following variables:

- Average price per megawatt.

- Total volume or quantity cleared.

- Total number of contracts cleared.

\section{Portland Results}

Figure 1 shows the performance of Portland bilateral market under different physical clearing rules.

- Average price per MW - The ascending order of clearing results in the highest average price per MW. However, only sets of contract size of 50 or lower clear the market and are feasible on the grid. This could be due to the fact that the small buyers are not able to negotiate a cheaper price with the suppliers due to the low volume. Under the descending order, prices are the highest for contract sets of 60 and higher. This is because when more contracts of high volume are sent to the grid, more congestion is created which can make some of the contracts infeasible on the grid. The contracts that do not clear on the grid cannot be honored in the market. This causes the buyers and sellers to negotiate another bilateral contract, this 
time may be with a more inefficient trader resulting in higher average clearing price. Surprisingly the random order clears the contracts at the cheapest average price.

- Total quantity cleared - For all contract sets, descending order clears more quantity than the random and ascending order. The descending order sends the biggest contracts first on the grid. Before the congestion starts to build up from additional contracts, a high volume gets cleared. Under ascending order, the smallest contracts go first resulting in lower quantity cleared. As additional contracts get loaded on to the grid, the spatial distribution of different source and sink nodes appear to cause more congestion and allow lower volume to clear. This shows that market driven grid dynamics play a crucial role in determining the performance of a clearing mechanism. The random order clears the least quantity. As the size of contract grouping increases we observe a downward trend in the quantity cleared for all three orders.

- Number of contracts cleared - Descending order of clearing leads to the highest average number of contracts cleared except for the contract sets of 10 and 20 when the ascending order clears more. For sets of size 60 and higher, ascending order does not clear any contracts, random order clears only a few and the descending order shows a cyclical behavior. The number of contracts cleared and the quantity cleared show consistent behavior overall. The lower quantity cleared is associated with the lower number of contracts cleared and vice versa. The random order performs the worst by this measure.

- Overall Trend - The overall trend of the graphs show that as contracts sets are made of higher and higher numbers, all four measures of performance for all orders of clearing, start to deteriorate. The total number of cleared contracts drops, the total volume drops and the average price per unit goes up. Overall the ascending order of clearing performs better for smaller sized contract sets. However, for higher sized contract sets, the descending order of clearing seems to beat other orders of clearing. The descending order is able to clear the most quantity and the highest number of contracts. For random and ascending orders, the quantity cleared and number of contracts cleared drop to almost zero after sets of size 60 .

\section{Chicago Results}

Figure 2 shows the performance of the Chicago bilateral market under different physical clearing rules.

- Average price per MW - For Chicago, there is no significant difference between the average price per MW under ascending and descending orders. It fluctuates around $\$ 40$ per MW for both these orders. For random order, the average price is slightly cheaper.

- Total quantity cleared - For almost all contract sizes the descending order clears the highest total quantity and the random order clears the lowest. For all three heuristics, the quantity cleared follows a downward trend as the contract group size increases.

- Number of contracts cleared - The descending order of clearing leads to the highest average number of contracts cleared for contract sets of size higher than 30. For smaller set sizes, the ascending order clears more contracts. The random order clears the least number of contracts for all set sizes. Again, there is an overall downward trend followed by the total number of contracts cleared as the size of contract sets increases.

- Overall Trend - The overall trend in the performance measures shows that as the size of the contract groups increases, the performance deteriorates. The total number of contracts cleared drops and the total volume drops as the set size increases. The average price on the other hand stays mostly steady. In general we find that the descending order of clearing performs better than the other orders of clearing and smaller size in contract groups results in better performance.

\section{Performance Comparison Between Portland and Chicago}

This section compares the results obtained for Portland and Chicago under different orders of clearing and contract set sizes.

- Similarities

- All performance measures, for all orders of clearing, deteriorate when the size of the contract groups increases.

- In general descending order beats other orders of clearing.

- The random order always results in lower quantity and contracts cleared for all sizes of contract sets.

- Overall trend of the measures is the same for both Portland and Chicago.

\section{- Differences}

- Although the total number of contracts cleared, volume cleared and average price show same structural properties for the two cities, quantitatively they are significantly different. For the city of Chicago, the number of contracts cleared and the volume cleared is much higher than it is for the city of Portland for all orders 
Average Price of Contract
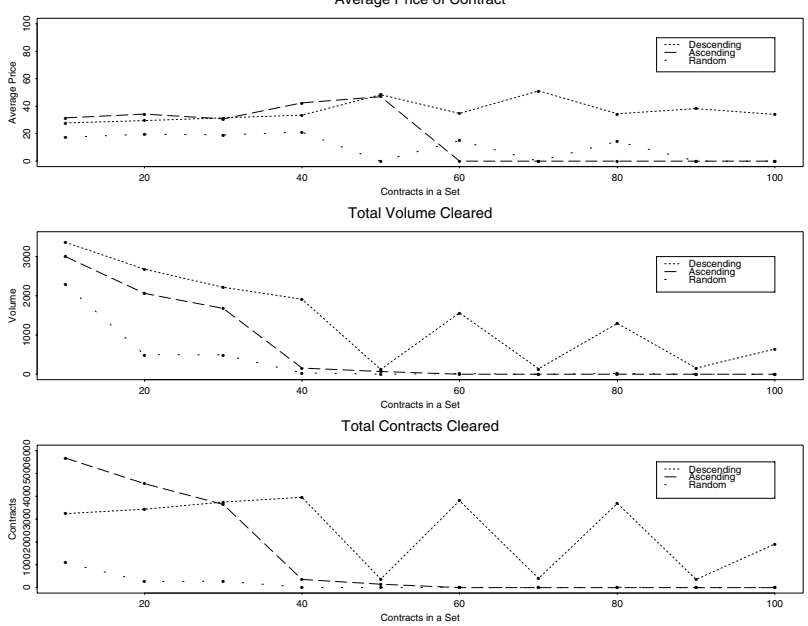

Fig. 1. Physical Clearing of Bilateral Contracts in Portland Performance under different sizes of contract sets and sorting orders

of clearing and contract sets. This is not surprising since the number of buyers, suppliers, locations and grid capacity is much higher in Chicago than in Portland.

- After contract sets of size 50 and higher, the quantity cleared and number of contracts cleared drop to zero in Portland whereas in Chicago they are substantially higher.

\section{- Implications}

- We performed the analysis on two different cities which have significantly different number of people, generators, buildings, substations and transmission capacity. Still the overall impact of the different physical clearing mechanisms on the market for Portland and Chicago is very similar. All performance measures follow similar trend in both the markets under different orders of clearing and contract set sizes. This is encouraging from the policy point of view since this suggests that once an optimal mechanism is determined, it may be uniformly applied to different regions and markets. Although more such studies for different regions are needed to confirm that similar results hold across other regions.

\section{Conclusions}

This paper studies the performance of several different physical clearing mechanisms in the electricity market. The physical clearing algorithms were chosen according to their ability to serve as a proxy for some of the well known policies such as minimum flow denied, maximum consumers served or fairness. Two cities, Portland and Chicago, are used for simulating the physical clearing algorithms. Our results show that, for both the cities,
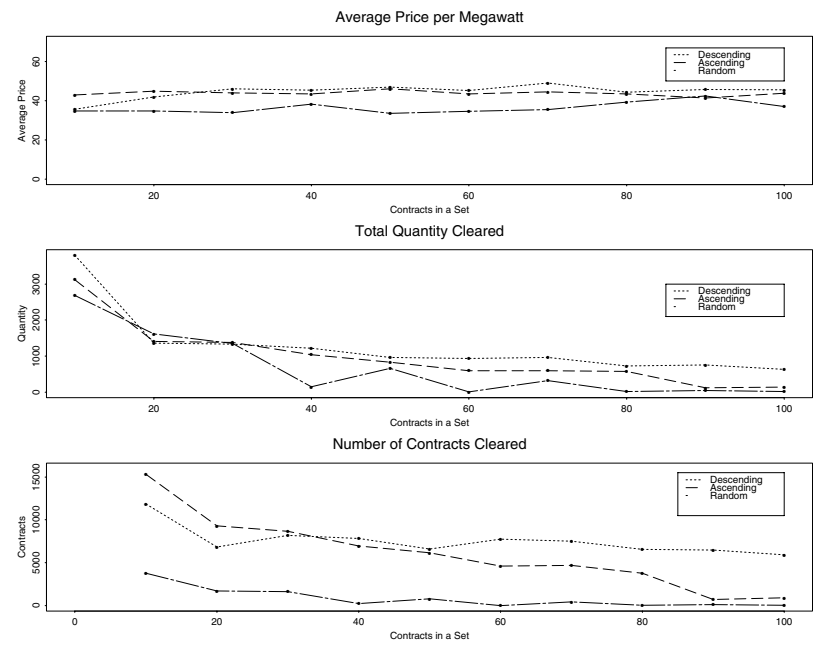

Fig. 2. Physical Clearing of Bilateral Contracts in Chicago Performance under different sizes of contract sets and sorting orders.

the performance deteriorates as the contract set size increases. This is true for all orders of clearing. In general, descending order beats other orders of clearing especially for higher contract set sizes. For the city of Chicago, the number of contracts cleared and the volume cleared is much higher than it is for the city of Portland for all orders of clearing and contract sets. This is to be expected since the number of people, locations and electrical grid capacity, all are much bigger in Chicago than in Portland. Except for the magnitude, the basic result from all physical clearing mechanisms is very much alike for both the cities. This could be good news to policy makers who can hope to get similar results in different regions from the same policy implementation. Note that this study ignores the electrical network resistances which implies that the results obtained here provide an upper bound to the results that would be obtained by using a resistive network. The network with resistance will only impose additional constraints on the physical clearing problem.

\section{REFERENCES}

[1] K. Atkins, C. Barrett, C. Homan, A. Marathe, M. Marathe, S. Thite, "Marketecture: A Simulation-Based Framework for Studying Experimental Deregulated Power Markets," submitted to Computational Economics, Los Alamos National Laboratory Technical Report, LAUR 04-2032.

[2] C. Barrett, D. Cook, V. Faber, G. Hicks, A. Marathe, M. Marathe, A. Srinivasan, Y. J. Sussmann, H. Thornquist, "Experimental Analysis of Bilateral-Contracts Clearing Mechanisms Arising in Deregulated Power Industry," Journal of Graph Algorithms and Applications, Volume 7, No. 1, 2003, pages 331.

[3] J. Bushnell, C. Knittel and F. Wolak, "Estimating the Opportunities for Market Power in a Deregulated Wisconsin Electricity Market," http: //www. customersfirst.org/marketpower_es.shtml. 
[4] S. Borenstein, J. Bushnell and F. Wolak, "Diagnosing Market Power in California's Restructured Wholesale Electricity Market," http: / / www. stanford.edu/ wolak (2000).

[5] J. Cardell, C. Hitt and W. W. Hogan, "Market Power and Strategic Interaction in Electricity Networks," Resource and Energy Economics, vol 19, 109-137, (1997).

[6] J. Carnes, "Why Use UTM Coordinates," http : / / www . maptools . com/UsingUTM/whyUTM . html (2001).

[7] M. J. Denton, S. J. Rassenti and V. L. Smith, "Spot Market Mechanism Design and Competitive Issues in Electric Power," Proceedings of the 31st International Conference on System Sciences: Restructuring of Electric Power Industry, (1998)

[8] L. J. Dowell, "How Diakoptics Works: An Example Power-Flow Problem," Los Alamos National Laboratory, LA-UR-99-6847, (1999).

[9] D. K. Gode and S. Sunder, "Allocative Efficiency of Markets with Zero-Intelligence Traders: Market as a Partial Substitute for Individual Rationality," Journal of Political Economy, vol. 101, no. 1, 119-137, (1993).

[10] J. E. Goodman and J. O'Rourke, "Handbook of Discrete and Computational Geometry," CRC Press, (1997).

[11] W. Hogan, "A Market Power Model with Strategic Interaction in Electricity Networks," Working Paper, Center for Business and Government, J.F. Kennedy School of Government, Harvard University (1997).

[12] I. Arciniegas, C. Barrett, A. Marathe, "Assessing the Efficiency of US Electricity Markets," Utilities Policy, Volume 11, Issue 2, June 2003, pages 75-86.

[13] J. Nicolaisen, V. Petrov and L. Tesfatsion, "Market Power and Efficiency in a Computational Electricity Market with Discriminatory Double-Auction Pricing," IEEE Transactions on Evolutionary Computation, vol. 5, No. 5, October 2001, pp. 546-560 (2001).

[14] C. R. Plot and S. Sunder, "Rational Expectations and the Aggregation of Diverse Information in Laboratory Security Markets," Econometrica, vol. 56, No. 5, 1085-1118, (1988).

[15] A. Rudkevich, M. Duckworth and R. Rosen, "Modeling Electricity pricing in a Deregulated Generation Industry: The Potential for Oligopoly Pricing in a Poolco," http: / / www. tellus.org/energy/, (1997).

[16] H. Shawky, A. Marathe and C. Barrett, "Estimating the Relationship between Electricity Futures and Spot Prices in the US,' Journal of Futures Market, Volume 23, Issue 10, October 2003 , pages 931-955.

[17] V. L. Smith, "An Experimental Study of Competitive Market Behavior,' The Journal of Political Economy, Vol 70, Issue 2 , 111-137, (1962).

[18] Urban Population Simulation Technology. Los Alamos National Laboratory. http://truchas.lanl.gov/ jmh/transims.html

[19] Public Service Commission of Utah, "Report to the Electrical Deregulation and Customer Choice Task Force," http: / /www.psc.state.ut.us/elec/Mktpwrpt.htm, (1998).

[20] USGS Mapping Applications Center, The Universal Transverse Mercator (UTM) Grid: Fact Sheet 077-01, http: / / mac.usgs.gov/mac/isb/pubs/factsheets / fs 07701 .html August (2001).

[21] A. M. Wildberger, "Issues associated with real time pricing. Unpublished Technical report," Electric Power Research Institute (EPRI), 1997.

[22] F. Wolak, "An Empirical Analysis of the Impact of Hedge Contracts on Bidding Behavior in a Competitive Electricity Market," http: / / www. stanford. edu/ wolak, (2000).

[23] J. Weiss, "Market Power Issues in a Restructuring of the Electricity Industry: An Experimental Investigation," http: / / www.ksg. harvard. edu/hepg/Jurgen2 . htm.

[24] C. Wolfram, "Measuring Duopoly Power in the British Electricity Spot Market," American Economic Review, 89, pages 805826, (1999). 